\title{
SOME COMMENTS ON FLARES AFTER MANY YEARS OF OBSERVATION
}

\author{
H. W. DODSON and E. R. HEDEMAN \\ McMath-Hulbert Observatory of The University of Michigan, 895 Lake Angelus Road, \\ Pontiac, Michigan, 48055, U.S.A.
}

\begin{abstract}
Ground based observations of flares are reviewed to seek implications for a flare build-up on either a long or a short time scale. Plots of flare frequency and importance for certain individual centers of activity suggest a possible crescendo in flare occurrence days and hours before the development of large and significant flares. The X-ray records follow the same pattern of apparent build-up. A possible dependence between successive major flares, as phases one and two of a single complex flare event, suggests that the time scale in which the total flare event takes place may show extreme variation.

Since all flares start as small features, there is a short term build-up in the optical records. The characteristics of this build up are not clear. The initial brightenings in a flare may or may not show a flash phase, and the rise to maximum may or may not be accompanied by filament activity. Flares rise to maximum $\mathrm{H} \alpha$ intensity at markedly different rates. Although most flares occur in centers of activity with well defined and often complex magnetic fields, certain large and relatively energetic flares have developed in centers of activity with apparently very simple circumstances.
\end{abstract}

\section{Introduction}

In preparation for the workshop on the flare build-up, we at the McMath-Hulbert Observatory reviewed some of the many ground based observations of past years and asked if these observations provide any information that can be interpreted as pertaining to this topic: a build-up on either a long or a short time scale.

A year ago, as part of the CINOF program, we asked if the very frequent occurrence of subflares in McMath plage 11926 on June 14, with their locations along a secondary neutral line, and at the apparent root of a complex filament system, might not constitute or indicate a build-up in the instabilities that finally led to the two more significant flares on the next day. Furthermore, for the 15th, we asked if the two larger flares of that day (now considered of importance 2) really were independent phenomena, or did the first, with its type II burst and subsequent major filament disappearance, constitute a form of build-up for the second, a large but faint flare, relatively far from spots, but with long enduring $\mathrm{X}$-ray, microwave, and type IV radiation. In our judgment, these are matters about which one can speculate and ask questions but for which it is very difficult to find convincing arguments.

\section{Frequency and Importance of Flares in Individual Centers of Activity}

\section{A. Centers with only moderate activity}

Let us consider first the pattern of the simple occurrence of flares and subflares in the center of activity with the CINOF flares of June 14 and 15, 1972 (McMath 
plage 11926). (Figure 1, part 3). Is the apparent increase in the frequency of small flares on the 13th and 14th prior to the greater events on the 15th a usual or unusual pattern in the flare history of moderately active centers of activity? According to our impression it is a frequent pattern but not invariable. We illustrate the situation with similar data for a small number of centers of activity which were only moderately active but which produced one or more apparently significant flares (Figure 1). For the first four regions a certain crescendo in flare occurrence seems present. For the last two cases the circumstances may be different. These latter cases refer to the occurrence of major $\mathrm{H} \alpha$ flares in regions with very small or no spots. The prior existence, during four or five days, of a very large, active filament over the associated plage should be noted.
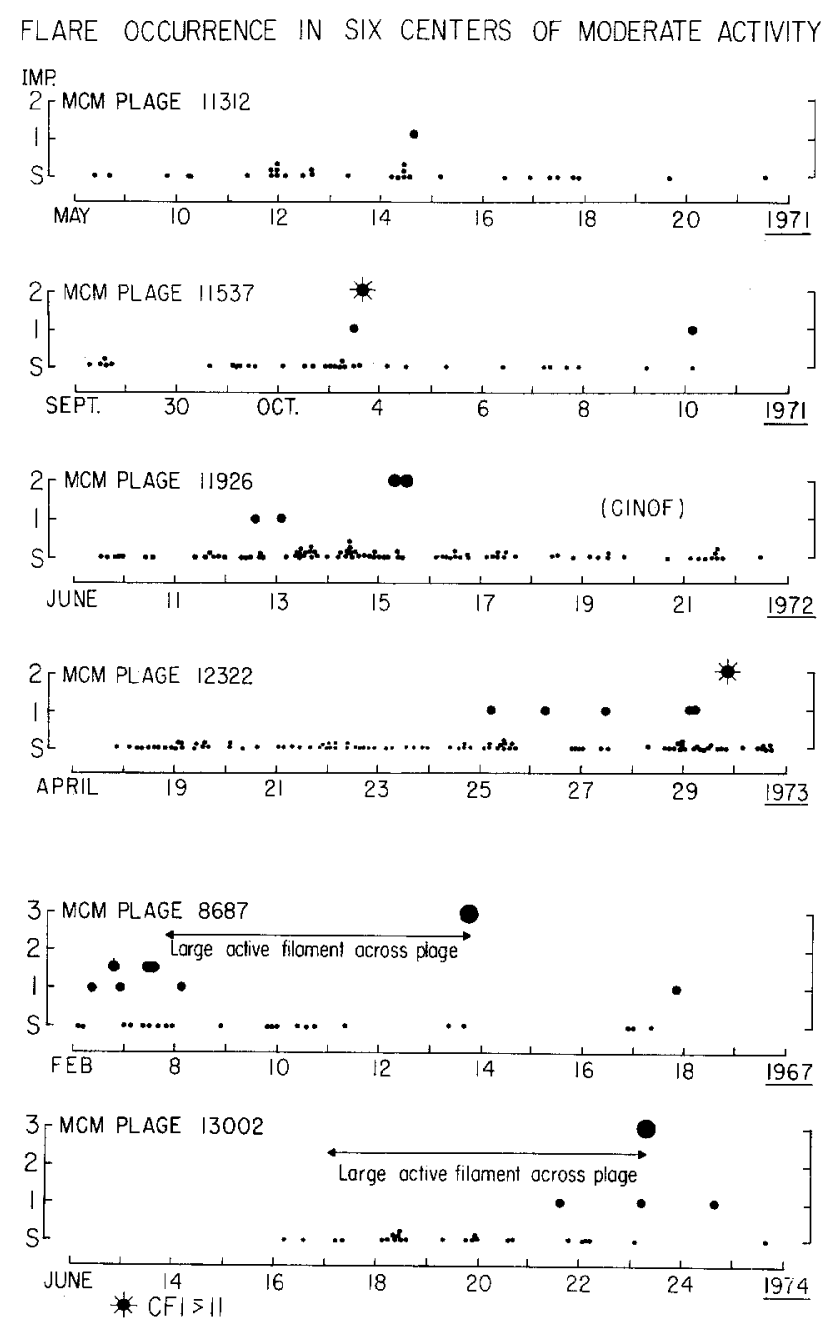

Fig. 1. Plot of flare occurrence in six centers of moderate activity. The size of the plotted dot increases with $\mathrm{H} \alpha$ flare importance. Spangles around the dot indicate that the comprehensive flare index was $\$ 11$. 
For all of the regions here shown the apparent buildup in the $H \alpha$ flare data was accompanied by time associated X-ray emission. The X-ray records for October 3, 1971 (case 2) make an apparent build-up over at least 10 hours especially clear (Figure 2).

\section{B. Centers with major Flare activity}

If we extend the data to some of the most flare-rich regions in cycles 19 and 20 , we find both similarities and differences (Figure 3). For the first four of the regions, all well known centers of activity, we see successive, apparent increases in the frequency and importance of flares and/or subflares in about a three day interval. For the last case, July 1974, however, the story seems different. Accordingly, if one chooses to do so, one can find in simple flare data a certain degree of apparent support for the concept of a long term build-up in the flare process, but one must always be mindful of the fact that there are numerous cases that do not conform to this pattern.

\section{The Double Flare}

Let us now consider further the possibility that two relatively significant events reported as separate flares, such as the two CINOF flares on June 15, 1972, may indeed be two phases of a single, complex physical phenomenon with the first event being a form of build-up for the second. About ten years ago we wrote an article on "Related Flares" (Dodson and Hedeman, 1966). At that time we focussed our attention on four events on September 18, 1957, for which the data suggested either four separate flares or two homologous flares each with two maxima. On the basis of both optical and radio frequency data we preferred the latter interpretation. The optical aspects of these flares reminded us of the differences between the early and late phases of many flares for which the development is apparently more rapid, and for which the phenomena are reported as only one flare event with either one or two maxima.

Over the years, we have even dared to ask if perchance the two great flares on July 16,1959 , did not possibly constitute phase one and phase two of a single very complex event on the Sun. Did the first flare, near $16^{\mathrm{h}}$, with its strong type II burst and $\mathrm{H} \alpha$ emission relatively far from spot umbrae, in anyway form part of the build-up for the greater flare at $\sim 21^{\mathrm{h}}$ with its emission close to and over umbrae, and its strong type IV emission (and type II "found" only after years of search)? (Figure 4.) If this type of relationship exists, it suggests that the time scale in which the total flare event takes place can show extreme variation from one case to another.

\section{The Short Term Build-up}

\section{A. H $\alpha$ RECORDS AND PHOTOMETRIC LIGHT CURVES}

With respect to the possibly very short term build-up of flares, we have tried to see if the $\mathrm{H} \alpha$ observations provide any information. The results of our efforts are 


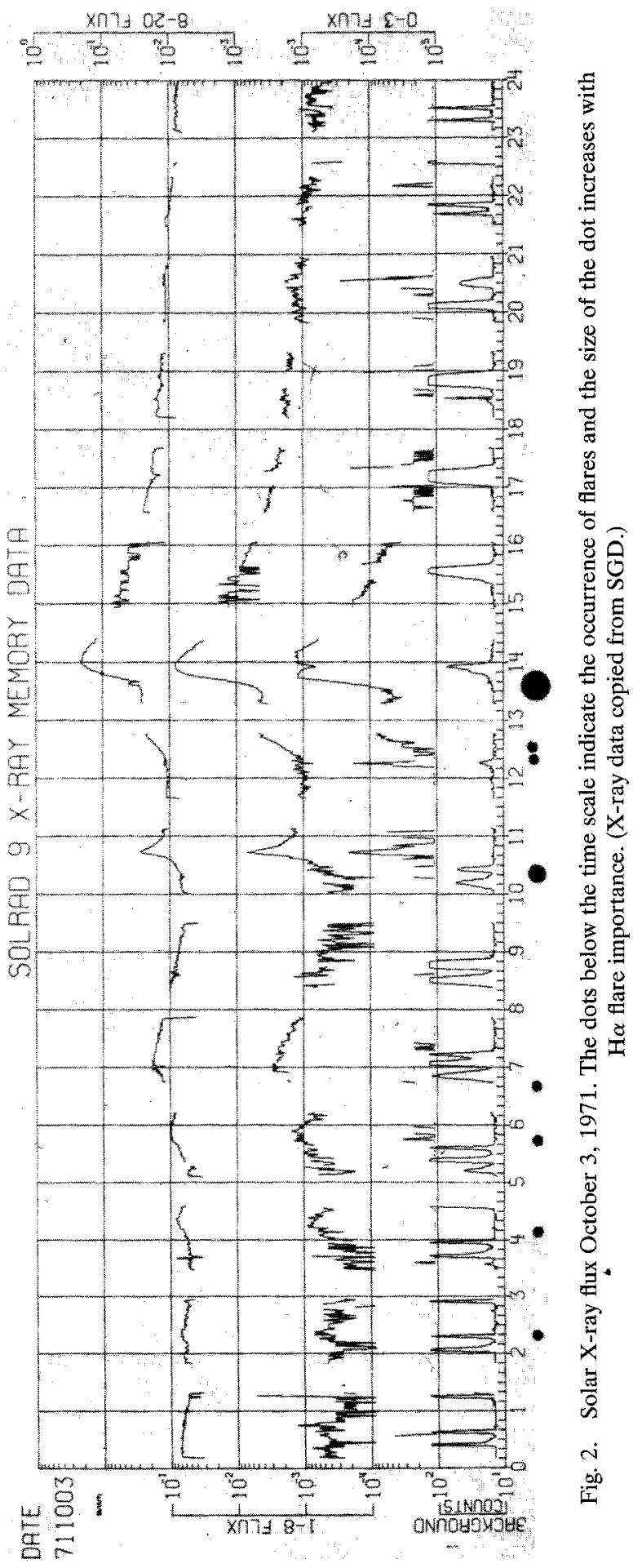




\section{FLARE OCCURRENCE IN FIVE CENTERS OF GREAT ACTIVITY}
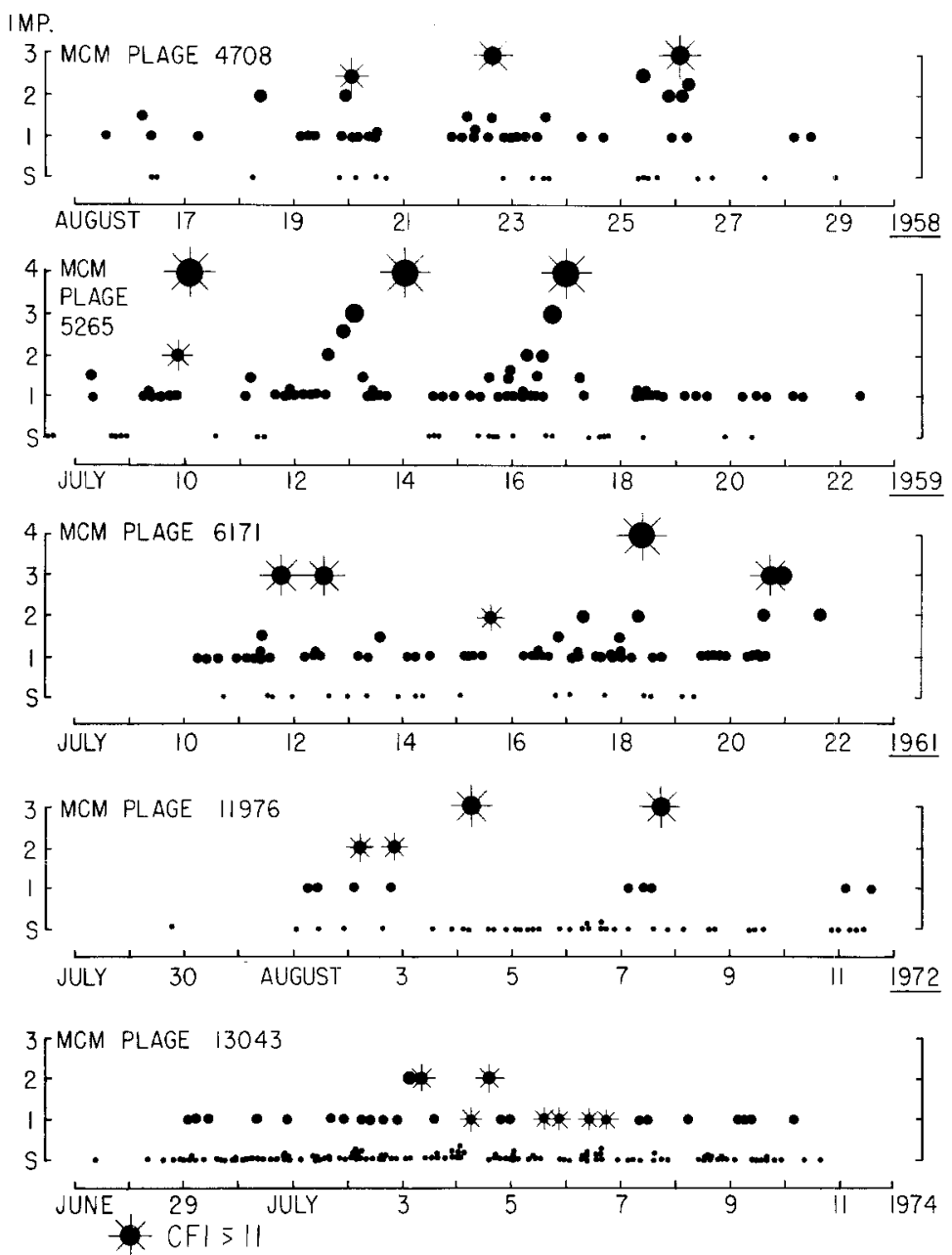

Fig. 3. Plot of flare occurrence in five centers of great activity. The size of the plotted dot increases with $\mathrm{H} \alpha$ flare importance. Spangles around the dot indicate that the comprehensive flare index was $\$ 11$. For the first three dates subflare data are seriously incomplete.

not impressive. For us it has proved difficult to recognize in optical data meaningful and general circumstances prior to or during the rise to maximum of flares.

We think it can be said with confidence that flares, no matter how large they may become at maximum, all start as small brightenings. In other words, there is a short term build-up in the optical data. The initial brightening of a flare, which is usually a small bright point near a neutral line, may or may not show a subsequent flash phase, and it may or may not become a portion of the most intense part of the fully developed flare. For example see data for the great flare of May 10, 1949 (Dodson, 1949). This flare began with the brightening of two points near a filament that traversed the plage. One of the points increased to 


\section{Comparison of Spot and Flares \\ 1959 July 16}
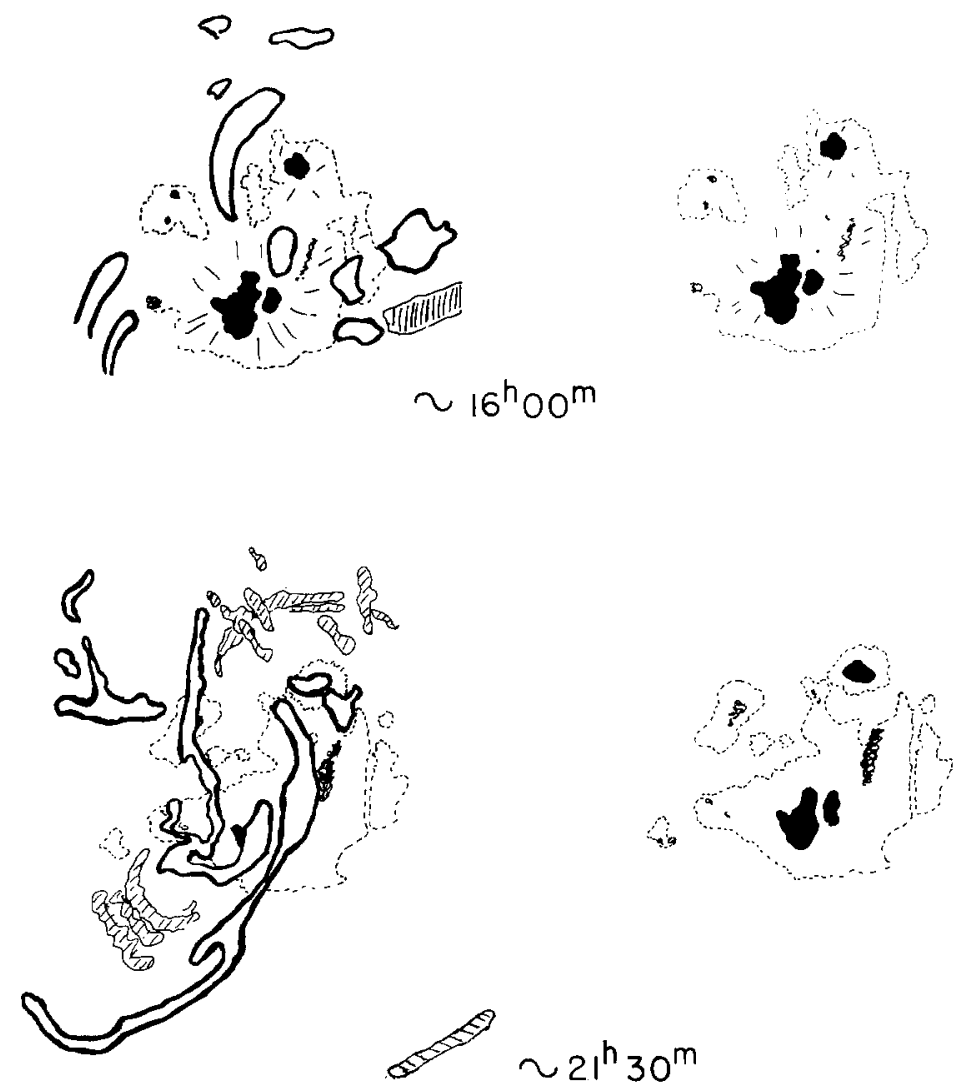

Fig. 4. Comparison of spot and flare locations for two important flares on July 16, 1959. At the right, the spot, umbra and penumbra, are shown as observed in the continuum. At the left, the $\mathrm{H} \alpha$ flare is shown by the full outline superposed over the spot. Hatched features indicate absorption markings.

maximum intensity very slowly. After some minutes, the second point showed a flash phase and more rapid increase. The brightest part of the flare was in another location and was directly over a spot umbra. It began its very sudden rise to maximum intensity about 15 minutes after the beginning of the flare event and continued as the brightest part of the flare for more than an hour.

Furthermore we can point out that the rate of increase of $\mathrm{H} \alpha$ intensity is not the same for all flares. Photometric measurements show at least three frequently observed rates of rise in $\mathrm{H} \alpha$ brightness (Dodson et al., 1956) (Figure 5). Increased $\mathrm{X}$-ray emission appears to accompany the slow as well as the rapidly increasing $\mathrm{H} \alpha$ radiation. Flares with slow $\mathrm{H} \alpha$ enhancement generally produce equally slow $\mathrm{X}$-ray enhancements. 


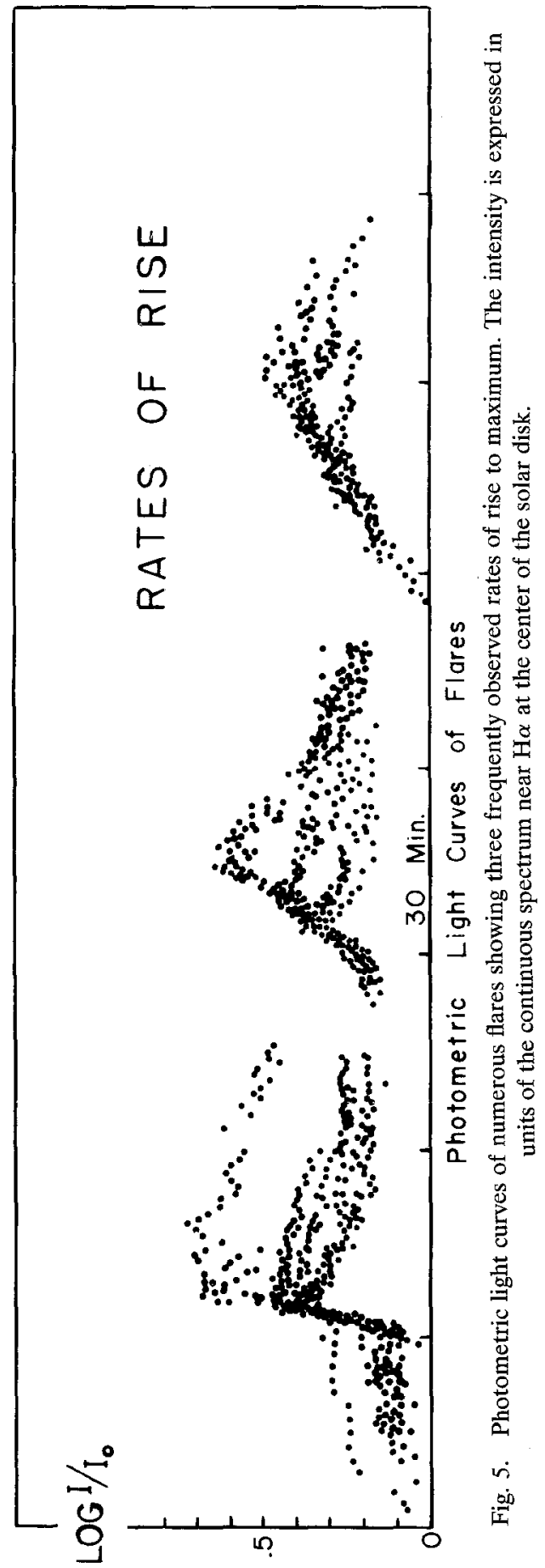




\section{B. Filament activation}

According to our observations, filament activation may or may not occur before and/or during the development phase of the flare. It may be significant that we have found it difficult to locate convincing examples of X-ray enhancements in the published graphs at the times of known filament activations unless there also were concomitant $\mathrm{H} \alpha$ brightenings. Nevertheless, the flash phase of the $\mathrm{H} \alpha$ flare frequently marks the spreading of $\mathrm{H} \alpha$ flare emission along the borders of previously existing filaments, either active or apparently quiescent.

\section{Observers' notes of flare deVELOPMENT}

For the most vivid impression of what can be observed in $\mathrm{H} \alpha$ during the early build-up of a flare, we recommend a reading of the notes of experienced observers who have been watching the Sun through a tunable $\mathrm{H} \alpha$ filter. Extensive comparison of such notes with published X-ray records for the times of phenomena near the central part of the solar disk, leaves us with a strong impression of very close rapport between the short term flare build-up as shown by increasing intensity and area of chromospheric brightenings, and the details of the X-ray records. For events near the limb, the observer's notes frequently provide the only clue to the fact that activity, possibly flare related, is taking place at or beyond the limb.

Sometimes at the beginning of a flare the situation on the Sun is very simple. At one minute the observer reports the solar disk and limb to be entirely calm. The next minute a small bright point has appeared and the $\mathrm{X}$-radiation starts to increase. At this time one is moved to ask if such a bright point with its still weak $\mathrm{X}$-ray and radio frequency emission will or will not develop into a significant flare? We feel that we do not know reliable answers to this important question. We often say in jest that the presence of an adjacent active filament or dark flocculus acts as a sort of "fertilizer" for the potential flare. Perhaps this circumstance plays some part in the short term flare build-up. On the other hand we have just pointed out that the initial small brightening appears to have a greater chance of becoming a significant flare if there has been an appreciable, general increase in flare frequency and magnitude during prior days or hours. These circumstances suggest that major flares may not be independent phenomena, and that their build-up, on both the long and short terms, is intimately related to a general increase in magnetic and/or coronal instabilities in the center of activity.

It is well known that when a center of activity includes large and complex spots, flares are usually frequent. However, major flares are not confined to this stage of development of a region. Important, very energetic flares often occur when the spots in a region, though still large, are well past maximum area and complexity. The rare cases of large flares in regions with only very small or no spots constantly remind us that the build-up for a large and moderately energetic flare can, in 
some instances, consist of apparently very simple solar circumstances. The evidence suggests that it is very easy for the Sun to produce a flare-but that it is extremely hard for us on Earth to understand with confidence the processes that take place.

\section{Acknowledgements}

The authors acknowledge with gratitude support from NOAA Contract No. 03-4022-94 and NASA Grant NGL-23-005-275 in the preparation of this study.

\section{References}

Dodson, H. W. and Hedeman, E. R.: 1966, Astrophys. J. 145, 224.

Dodson, H. W.: 1949, Astrophys. J. 110, 382.

Dodson, H. W., Hedeman, E. R. and McMath, R. R.: 1956, Astrophys. J. Supplement Number 20, 241.

\section{Discussion}

DRYER: With reference to your figure which showed the $\mathrm{H} \alpha$ rise time intensity enhancements some fast, some slow - is it possible to make some associations, vis-a-vis radio observations (i.e., with types III, II, and/or IV)?

DoDson: It has been our experience that the rate of increase of $\mathrm{H} \alpha$ intensity does not provide a reliable guide to the type of dynamic spectrum feature that occurs at radio frequencies. For example, the major flares in regions with small or no spots, with their generally slow rate of rise to maximum, are variously accompanied by radio bursts of Types II, III and IV, as is also the case for flares with much more rapid increases in $\mathrm{H} \alpha$.

ŠvESTKA: Some years ago you published a paper stating that all "spotless" flares follow a filament activation. Some people, e.g., D. Rust, believe that all flares are preceded by filament activation and that the filament disruption triggers the flare. We certainly do not see the filaments prior to flares in very young active regions, where the filament did not yet become visible. But I want to ask you: are there flares in older active regions (where filaments are present) which are not preceded by a filament activation?

DoDson: For decades we at the McMath-Hulbert Observatory have been strong advocates of a close association between filaments and flares. Nonetheless, for some flares our observing records with tunable $\mathrm{H} \alpha$ filters, for several hours prior to and during the rise to $\mathrm{H} \alpha$ maximum, provide no evidence of filament activation recognizable in our telescopes. We, therefore, in our foregoing paper felt obliged to say that the short term build-up of flares "may or may not" include filament activation. Further study of this matter seems to require agreement on the size and extent of what is meant by "filament activation" and the prior time interval in which such phenomena would be considered relevant to a flare.

SHEELEY: Regarding the "invisible structures" now visible in the X-ray and XUV part of the spectrum. Our access to this region of the operations gives us (in effect) a new pair of eyes. The excitement of seeing these new solar features must be similar to that experienced by solar observers soon after the invention of the spectroheliograph when many new phenomena such as filaments which are not visible in white light became visible.

Dodson: Yes; even the change from the $\mathrm{Ca}^{+} \mathrm{K}$ line to the $\mathrm{H} \alpha$ revealed many exciting new features. 